\title{
EBIC and DLTS characterization of pure Si crystals
}

\author{
E B YAKIMOV \\ Institute of Microelectronics Technology RAS, Chernogolovka 142432, Russia
}

\begin{abstract}
Possibilities of measurements of low defect concentration in $\mathrm{Si}$ by the electrical methods are discussed. The problems arising in such measurements are illustrated by measurements of iron concentration in $\mathrm{Si}$. It is demonstrated that gold diffusion experiments can be used for revealing and study of some electrically inactive defects. Possibility of nondestructive reconstruction of defect depth profiles by DLTS and using the profile obtained for understanding the defect nature is illustrated by the results of hydrogen and hydrogen related defect investigations. EBIC investigations of dislocations are shown to be a rather sensitive method for revealing recombination defects.
\end{abstract}

Keywords. Defect; impurity; Si; DLTS.

\section{Introduction}

With decreasing dimensions and increasing complexity of highly integrated circuits, the tolerable concentrations of detrimental impurities in Si wafers have to shrink considerably. In particular, a contamination due to transition metals with their high diffusivity is critical for all $\mathrm{Si}$ technology processes. In the recent $\mathrm{Si}$ wafers the concentration of transition metals, e.g. iron, should not exceed $10^{10} \mathrm{~cm}^{-3}$. Therefore, a question arises about the methods allowing measurements of such ultra low impurity concentrations. For electrically active defects the concentration as low as $10^{10}-10^{11} \mathrm{~cm}^{-3}$ can be deduced in processed silicon wafers from measurements of electrical characteristics, such as minority carrier lifetime or diffusion length. Study of deep level centre concentration by the deep level transient spectroscopy (DLTS) method also provides the comparable sensitivity but only in low-doped crystals. However, for the successful use of such methods for the measurements of impurity concentration some material science problems should be solved. It should also be stressed that the DLTS measurements can be used also for revealing electrically neutral defects if some indirect approaches are used. The present paper illustrates problems arising under measurements of low defect concentration in Si by electrical methods and the possible ways for their solution. The possibility of indirect measurements of electrically inactive defects by the electrical methods is demonstrated.

\section{Determination of iron concentration in $\mathbf{S i}$}

Iron is considered to be the most important impurity in $\mathrm{Si}$ technology since it is a main constituent of the techno-

yakimov@ipmt-hpm.ac.ru logical equipment and a common contaminant in chemicals (Graff 1995). Apart from its role as a recombinationgeneration centre it is extremely detrimental in metalon-insulator (MOS) technology. Therefore, many investigations have been devoted to the properties of iron in $\mathrm{Si}$ and to the development of technological processes in order to suppress its effect on electrical properties of devices (Istratov et al 1999). Now it is well known that if the wafer is slowly cooled after high temperature annealing about all iron is precipitated and could not be revealed by the electrical methods. Thus, to obtain the iron concentration by DLTS or lifetime measurements, first of all, it is necessary to transform it in the electrically active state. It could be done, for example, by quenching the sample under study from temperature, at which the iron solubility limit is higher than its total concentration. Thus, higher the iron content, the higher temperature should be used for its activation. After quenching iron atoms present in $\mathrm{Si}$ in the interstitial position and in $p$-Si they could form pairs with boron even at room temperature. The duration of this process depends on boron concentration and could vary from a few hours to a few days. In both the states iron is electrically active and can be revealed by DLTS (figure 1). The FeB pairs are unstable under illumination or annealing at $200-250^{\circ} \mathrm{C}$. Therefore, quenching from this temperature could return iron in the interstitial position (figure 1) and it again could form FeB pairs at room temperature. The sensitivity of DLTS measurements depends on the doping level of sample under study. Nevertheless, in Si doped with boron to the concentration of about $10^{15} \mathrm{~cm}^{-3}$ iron in a concentration of $10^{11} \mathrm{~cm}^{-3}$ is well revealed by DLTS (figure 2).

The excess carrier lifetime (or diffusion length) measurements are even more sensitive to the iron concentration. But while DLTS allows to obtain the iron concentration directly, in the lifetime measurements it is necessary to identify iron, separate the iron effect from that of other 
recombination centres and to know the conversion factor between the lifetime value and iron concentration. The well known procedure for this consists in lifetime measurements with $\mathrm{Fe}$ in the interstitial position and in $\mathrm{FeB}$ pairs and then the iron concentration could be calculated using the expression (Lagowski et al 1992)

$$
[\mathrm{Fe}]=K\left[\frac{1}{\tau_{\mathrm{Fe}_{\mathrm{i}}}}-\frac{1}{\tau_{\mathrm{FeB}}}\right],
$$

where $K$ is the conversion factor. Unfortunately, the $K$ value is very sensitive to the excitation level used under measurements (Rommel et al 2002), i.e. to the relation $\Delta p / p_{0}$, where $\Delta p$ and $p_{0}$ are the excess and equilibrium carrier concentrations, respectively. Therefore, for the correct determination of iron concentration the $K$ dependence on the excitation level should be known. The procedure allowing the determination of this dependence based on lifetime measurements in $\mathrm{Si}$ with different boron concentrations contaminated with iron was proposed in Palais et al (2002). It was seen (figure 3) that $K$ strongly increases (sensitivity to iron concentration decreases) with the excitation level and at high excitation level it could even change its sign. Such procedure allows not only to measure $K\left(\Delta p / p_{0}\right)$ dependence but also to obtain

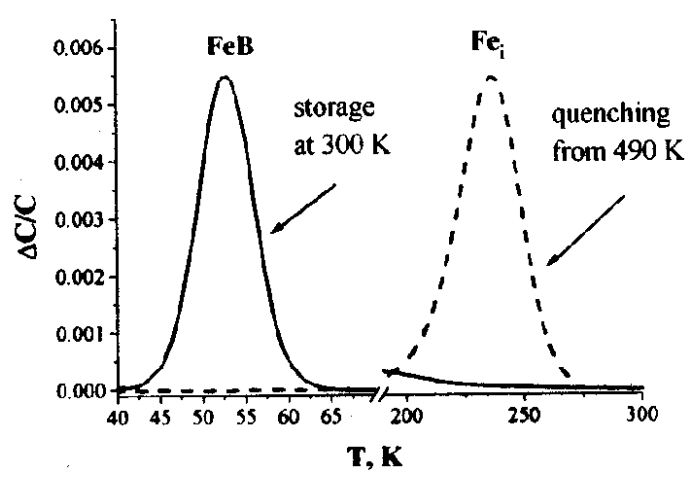

Figure 1. DLTS spectra of iron in different states.

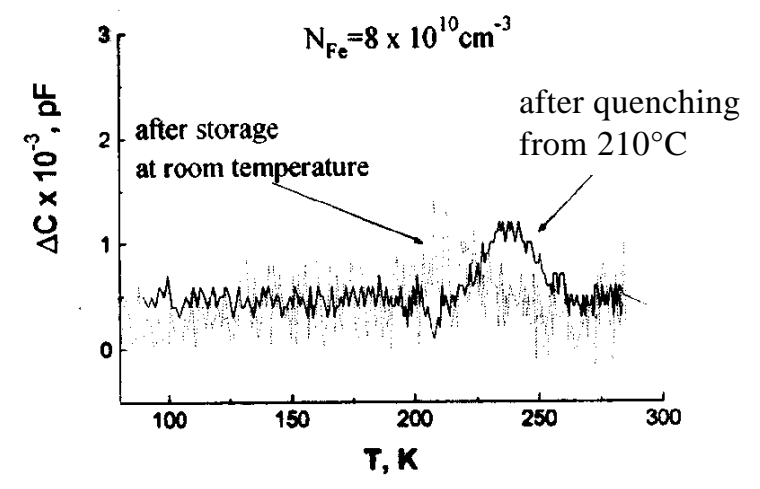

Figure 2. DLTS spectrum of interstitial iron in $\mathrm{Si}$ with low $\mathrm{Fe}$ concentration. the electron and hole capture cross-section values for $\mathrm{Fe}$ and $\mathrm{FeB}$ by fitting the experimental data (figure 3 ). In turn, the knowledge of capture cross-section values allows to calculate $K$ for any excitation and doping levels using the expression given in Shockley and Read (1952) and Hall (1952).

\section{Study of hydrogen related effects in $\mathrm{Si}$}

One of the advantages of DLTS technique is the possibility of nondestructive reconstruction of defect depth distribution. Such reconstruction could be used for the study of depth distribution of defects in a wafer but in some cases it helps to reveal the nature of defects under study. Such application of DLTS measurements can be illustrated by the results of study of hydrogen interaction with other point defects in $\mathrm{Si}$. If hydrogen is introduced in $\mathrm{Si}$ at room temperature under wet chemical etching (WCE), its concentration is rather low and it penetrates into $\mathrm{Si}$ to a depth depending on defect concentration and usually varying in the range from $0 \cdot 1-10$. Hydrogen penetration in $\mathrm{Si}$ under such process can be monitored by the electrical methods due to a passivation of shallow impurities (Feklisova et al 2002). In figure 4 depth profiles of electrically active boron in $p$-Si after WCE reconstructed from the $C-V$ measurements together with simulated dependences are presented. In such samples practically all penetrated hydrogen is captured by boron, therefore, a difference between the bulk boron concentration and that near surface is equal to hydrogen concentration. If the sample is annealed at $340-380 \mathrm{~K}$ with reversed bias applied to the Schottky barrier, hydrogen-boron pairs are destroyed and the positively charged hydrogen drifts in the electric field deeper into the sample to the depletion region edge. In this region free hydrogen could interact with deep-level centres and partly could be recaptured by boron. A study of depth profiles of defects created due to hydrogen interaction with metal impurities or radiation

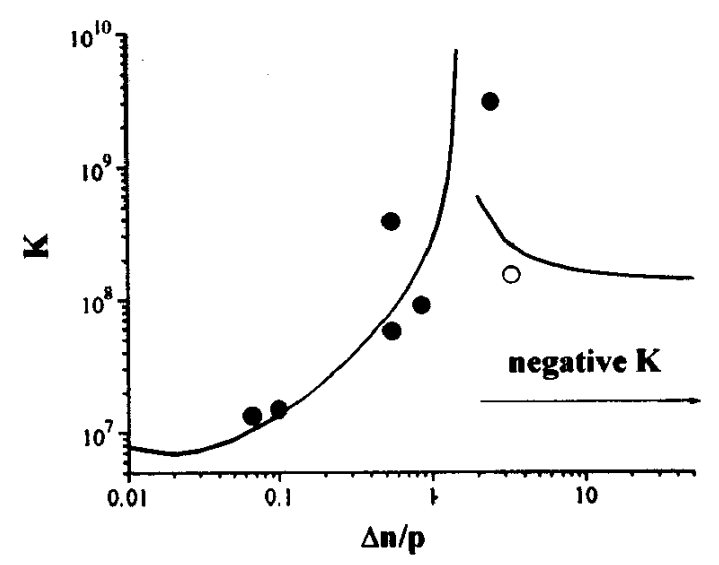

Figure 3. Measured (symbols) and calculated (lines) dependences of $K$ on excitation. 
defects after WCE and reverse bias annealing and a comparison with the boron profile helps to clarify the nature of defect revealed and to prove their relation to hydrogen (Feklisova et al 1999, 2000; Sachse et al 1999). An example of such study is presented in figure 5, where the depth profiles of electrically active boron and defects revealed in electron irradiated $\mathrm{Si}$ after reverse bias annealing are presented. It is seen that the effective boron concentration decreases at a depth of about $4 \mu \mathrm{m}$ due to boron passivation. That means that the hydrogen concentration at such annealing has a peak at this depth. At the same depth a decrease in concentration of divacancies, $\mathrm{C}_{\mathrm{s}} \mathrm{C}_{\mathrm{i}}$ and $\mathrm{C}_{\mathrm{i}} \mathrm{O}_{\mathrm{i}}$ pairs, is also observed that means that all these defects are also interacted with hydrogen. At the same time the concentration of $\mathrm{H} 3$ and $\mathrm{H} 4$ centres has a maximum at this depth that allows to associate them with hydrogen related defects formed as a result of hydrogen interaction with radiation defects.

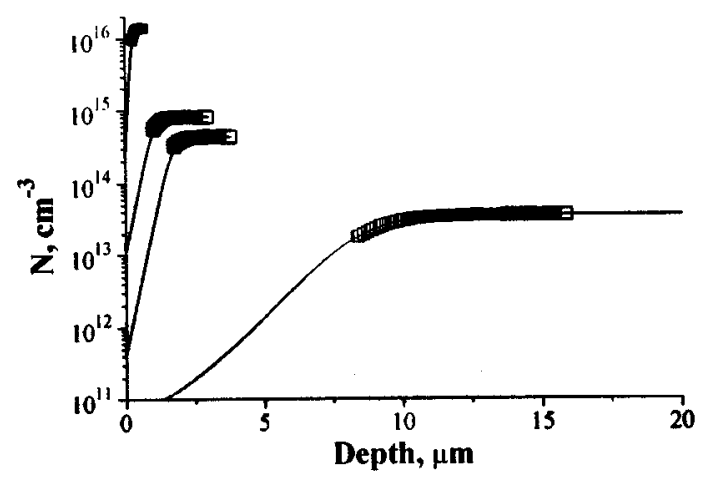

Figure 4. Profiles of electrically active boron in $p$-type $\mathrm{Si}$ with different doping after WCE.

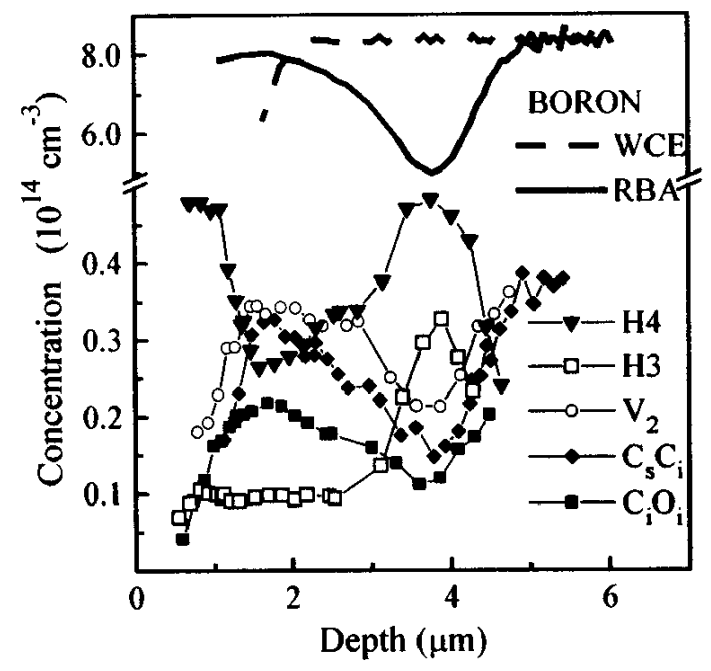

Figure 5. Depth profiles of boron and deep-level centre concentrations in $p$-type irradiated FZ-Si after WCE and subsequent reverse bias annealing at $100^{\circ} \mathrm{C}$.

\section{Evaluation of electrically inactive defect concentrations in $\mathrm{Si}$ by indirect methods}

The electrically inactive defects cannot be studied by the electrical methods directly. But sometimes it could be done indirectly by studying the interaction of these neutral defects with electrically active defects. One example of such measurements is presented in figure 6 , where the effective boron profiles in two $\mathrm{Si}$ samples with the same boron but different oxygen concentrations are presented. The difference between these two profiles could be associated with the defects containing oxygen and hydrogen. These defects are neutral, nevertheless, their depth profile can be reconstructed from the electrical measurements.

The other indirect methods for evaluation of small defect concentrations in $\mathrm{Si}$ are illustrated by the experiments with gold diffusion and dislocation related gettering. It is well known that gold in defect-free $\mathrm{Si}$ diffuses very quickly over interstitial sites and then it is transformed into electrically active substitutional sites via the kick-out mechanism (Frank et al 1984; Stolwijk et al 1986; Zimmermann and Ryssel 1992). Therefore, the concentration of substitutional gold is very sensitive to the concentration and distribution of traps for self-interstitials and to the self-interstitial generation processes. It was demonstrated that gold diffusion study can be used as a rather sensitive tool for the defect characterization when the substitutional gold concentration was measured by the DLTS technique. For such investigations first of all, the temperature dependence of gold concentration in defect-free $\mathrm{Si}$ was obtained in the temperature range from $700-1000^{\circ} \mathrm{C}$ (Feklisova and Yakimov 2004). Such dependence for normalized gold concentration

$$
A(T)=N_{\mathrm{Au}} d / 2(\pi t)^{1 / 2},
$$

where $d$ is the sample thickness and $t$ the diffusion duration, is presented in figure 7 . Then the deviation of gold concentration from this reference dependence in some samples can be considered as an indication of presence of

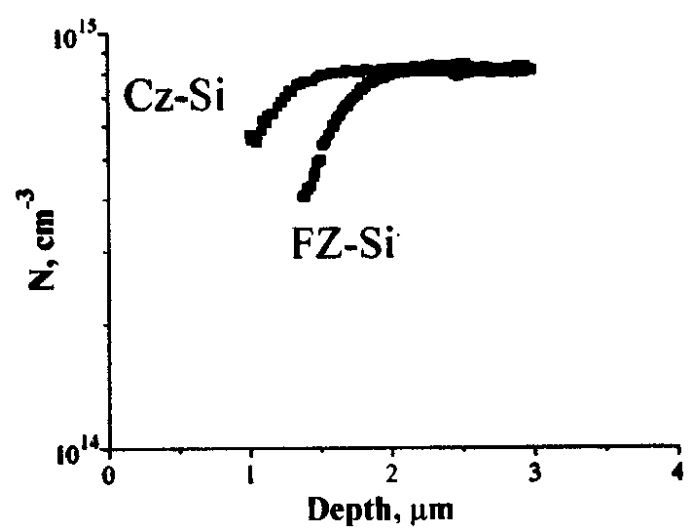

Figure 6. Depth profiles of electrically active boron in $\mathrm{Cz}$ and FZ $p$-type Si after WCE. 


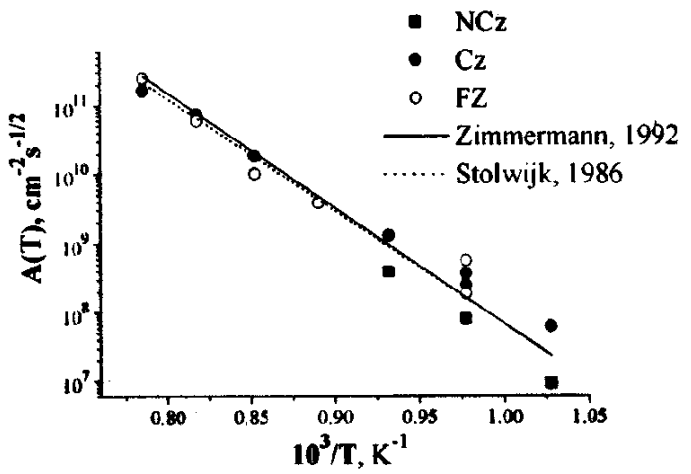

Figure 7. Normalized $\mathrm{Au}_{s}$ concentration as a function of diffusion temperature.

sinks for self-interstitials (or vacancy type defects) or by self-interstitial generation during gold diffusion annealing. The first type of behaviour can be illustrated by the dislocation effect on the substitutional gold concentration (Yakimov et al 1995). It was shown that dislocations play a role of effective self-interstitial sinks that leads to an essential increase of gold concentration as compared with a reference dislocation-free sample.

The application of gold diffusion measurements for the characterization of defect introduced under ion implantation is illustrated by the experiments carried out on $\mathrm{H}$ and He implanted samples, in which nanocavities are produced (Perichaud et al 2001). Such procedure could be very promising for the development of new gettering strategy consistent with the low thermal budget required for the Giga-scale integration. In these experiments unlimited gold source is formed on the back surface, nevertheless, the $\mathrm{Au}_{s}$ profile is essentially modified by nanocavities (figure 8). It is seen that nanocavities effect the gold distribution up to a distance of about $60 \mu \mathrm{m}$ from the nanocavity layer. The analyses of results obtained show that the capture of $\mathrm{Au}_{i}$ by nanocavities and selfinterstitial generation probably affect the formation of depth profile observed. Nitrogen effect on the grown-in defects in Si can be also studied using the gold diffusion experiments. Thus, as shown by Feklisova and Yakimov (2004), concentration of vacancy type defects playing a role of effective gold interstitial traps and their radial distribution can be studied by the gold diffusion experiments. It was shown that such defects are formed in floating zone (FZ) Si doped with nitrogen up to a concentration of about $10^{14} \mathrm{~cm}^{-3}$ while no such defects were revealed in FZ Si without nitrogen.

Dislocations in Si crystals are rather effective impurity getters. They could getter detrimental metal impurities and as a result, their recombination activity is increased. The recombination strength of dislocation and thus their contamination could be controlled by the electron beam induced current (EBIC) method (Yakimov 1996). The EBIC contrast of clean dislocation in $\mathrm{Si}$ is very low and

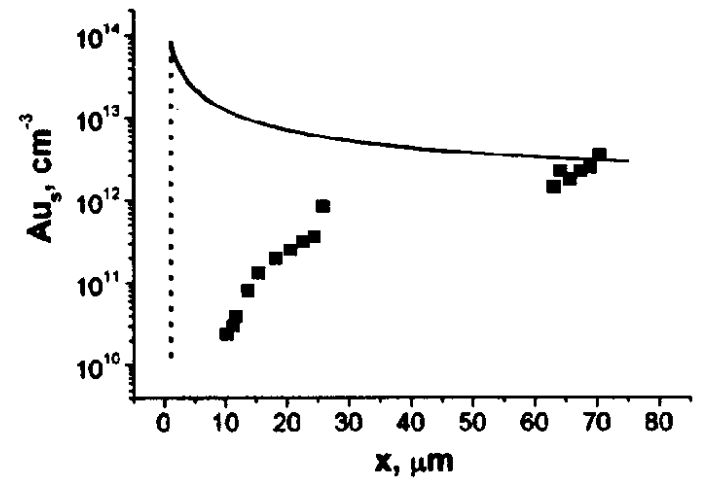

Figure 8. $\mathrm{Au}_{s}$ depth distribution after diffusion at $750^{\circ} \mathrm{C}$ for $1 \mathrm{~h}$ in $\mathrm{Si}$ with nanocavities produced by $\mathrm{He}$ implantation. The nanocavity depth and $\mathrm{Au}_{s}$ distribution for defect-free wafer are indicated by dotted and solid lines, respectively.

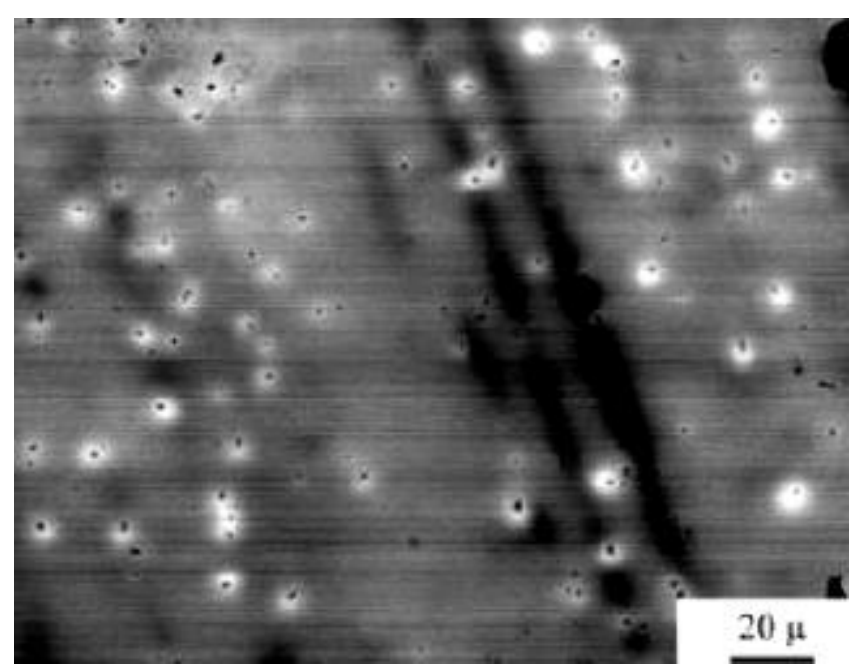

Figure 9. EBIC image of dislocated $\mathrm{Si}$ after gold diffusion at $1000^{\circ} \mathrm{C}$ for $2 \mathrm{~h}$

could not be detected (Kittler and Seifert 1996). But in contaminated Si the contrast increases and could be easily measured. That allows indirect control of the purity of samples by a study of individual dislocation EBIC contrast. The EBIC image of $\mathrm{Si}$ with dislocations contaminated with gold is presented in figure 9. It is seen that not only dislocations can be revealed as black points but all dislocations are surrounded with bright halos. These bright regions are formed due to gold gettering to dislocations. Decrease in gold concentration leads to a local minority carrier lifetime increase and therefore, to a bright EBIC contrast.

\section{Conclusions}

Thus, the prospects and limitations of electrical methods for pure Si characterization is discussed. The experimen- 
tal results demonstrating the sensitivity of these methods, their possibilities for revealing electrically active and inactive defects and estimating concentration of these defects are presented.

\section{References}

Feklisova O V and Yakimov E B 2004 Solid state phenomena (eds) H Richter and M Kittler (Uetikon, Switzerland: Scitec. Publ. Ltd.) 95-96, p. 495

Feklisova O, Yarykin N, Yakimov Eu and Weber J 1999 Physica B: Condens. Matter 231273

Feklisova O V, Parakhonsky A L, Yakimov E B and Weber J 2000 Mater. Sci. Eng. B71 268

Feklisova O V, Yakimov E B and Yarykin N A 2002 Semiconductors $\mathbf{3 6} 282$

Frank W, Gösele U, Mehrer H and Seeger A 1984 Diffusion in crystalline solids (Orlando: Academic Press Inc.) p. 64

Graff K 1995 Metal impurities in silicon-device fabrication (Berlin: Springer-Verlag) p. 66

Hall R N 1952 Phys. Rev. 87387
Istratov A A, Hieslmair H and Weber E R 1999 Appl. Phys. A69 13

Kittler M and Seifert W 1996 Mater. Sci. Eng. B42 8

Lagowski J, Edelman P, Dexter M and Henley W 1992 Semicond. Sci. Technol. A7 185

Palais O, Yakimov E and Martinuzzi S 2002 Mater. Sci. Eng. B91-92 216

Perichaud I, Yakimov E, Martinuzzi S and Dubois C $2001 \mathrm{~J}$. Appl. Phys. 902806

Rommel M, Zoth G, Ulrich M and Ryssel H 2002 Solid state phenomena (eds) V Raineri et al (Switzerland: Scitec Publ. Ltd) 82-84, p. 373

Sachse J-U, Sveinbjörnsson E Ö, Yarykin N and Weber J 1999 Mater. Sci. Eng. B58 134

Shockley W and Read W T 1952 Phys. Rev. 87835

Stolwijk N A, Hölzl B J, Frank W, Weber E R and Mehrer H 1986 Appl. Phys. A39 37

Yakimov E, Mariani G and Pichaud B 1995 J. Appl. Phys. 78 1495

Yakimov E B 1996 Phys. Low-Dimen. Struct. 1/2 77

Zimmermann H and Ryssel H 1992 Appl. Phys. A55 121 